\title{
Origin and Evolution of Prebiotic Organic Matter as Inferred from the Tagish Lake Meteorite
}

Christopher D. K. Herd ${ }^{1 *}$, Alexandra Blinova ${ }^{1}$, Danielle N. Simkus ${ }^{1}$, Yongsong Huang ${ }^{2}$, Rafael Tarozo $^{2}$, Conel M. O'D. Alexander ${ }^{3}$, Frank Gyngard ${ }^{3}$, Larry R. Nittler ${ }^{3}$, George D. Cody ${ }^{4}$, Marilyn L. Fogel ${ }^{4}$, Yoko Kebukawa ${ }^{4}$, A. L. D. Kilcoyne ${ }^{5}$, Robert W. Hilts ${ }^{6}$, Greg F. Slater ${ }^{7}$, Daniel P. Glavin ${ }^{8}$, Jason P. Dworkin ${ }^{8}$, Michael P. Callahan ${ }^{8}$, Jamie E. Elsila ${ }^{8}$, Bradley T. De Gregorio $^{9,10}$, Rhonda M. Stroud ${ }^{10}$

1 - Department of Earth and Atmospheric Sciences, University of Alberta, Edmonton, AB, T6G 2E3, Canada

2 - Department of Geological Sciences, Brown University, 324 Brook Street, Providence, RI 02912

3 - Department of Terrestrial Magnetism, 5241 Broad Branch Road, Carnegie Institution of Washington, Washington, DC, 20015

4 - Geophysical Laboratory, 5251 Broad Branch Road, Carnegie Institution of Washington, Washington, DC, 20015

5 - Advanced Light Source, Lawrence Berkeley Laboratory, Berkeley, CA, 94720.

6 - Department of Physical Sciences, Grant MacEwan University, Edmonton, AB, T5J 4S2, Canada

7 - School of Geography and Earth Sciences, McMaster University, Hamilton, ON, L8S 4K1, Canada

8 - NASA Goddard Space Flight Center, Greenbelt, MD 20771

9 - NASA Johnson Space Center/ESCG, Houston, TX 77058

10 - Naval Research Laboratory, 4555 Overlook Ave. SW, Washington, DC, 20375

*To whom correspondence should be addressed. E-mail: herd@ualberta.ca 
$\underline{\text { Abstract }}$

The complex suite of organic materials in carbonaceous chondrite meteorites probably originally formed in the interstellar medium and/or the solar protoplanetary disk, but was subsequently modified in the meteorites' asteroidal parent bodies. The mechanisms of formation and modification are still very poorly understood. We carried out a systematic study of variations in the mineralogy, petrology, and soluble and insoluble organic matter in distinct fragments of the Tagish Lake meteorite. The variations correlate with indicators of parent body aqueous alteration and at least some molecules of pre-biotic importance formed during the alteration.

Carbonaceous chondrite meteorites are samples of $\mathrm{km}$-sized primitive asteroids that preserve to varying degrees the initial solid components of the solar protoplanetary disk (or nebula (1). As such, these meteorites are samples of the material that took part in planet formation nearly 4.6 billion years ago. The chondrites also preserve a record of the processes that occurred in their asteroid parent bodies, such as thermal metamorphism, aqueous alteration and impact brecciation $(l)$. Organic matter composes up to several wt $\%$ of carbonaceous chondrites and includes macromolecular material and a variety of simpler molecules (2) that are generally referred to as insoluble (IOM) and soluble organic matter (SOM), respectively, because of their relative solubilities in typical solvents (e.g., 3, 4). Organic matter in carbonaceous chondrites shares characteristics with material from other primitive extraterrestrial samples, including interplanetary dust particles (IDPs), samples of comet 81P/Wild-2 (5-6) and some Antarctic micrometeorites (7). The common features of IOM from carbonaceous chondrites and comets suggest that there was a common source of such organic matter - the outer solar nebula and/or the interstellar medium (ISM) - and that the diversity of organic matter in meteorites is the result of variable degrees of parent body modification ( 8 ).

Earth's carbon was provided by accretion of early solar system solids. The accretion of meteorites, as well as other asteroidal and cometary material by the early Earth may have been a source of intact organic matter that was necessary for the advent of life (9). Carbonaceous chondrite SOM includes molecules of pre-biotic interest such as amino acids, nucleobases, monocarboxylic acids (MCAs), sugars, and polycyclic aromatic hydrocarbons (e.g., 3). 
Some of these compounds may be the result of hydrothermal alteration of IOM in the meteorite parent bodies (10-12), but which formed in this manner is an open question.

Here we report on IOM and SOM in several individual stones of the Tagish Lake meteorite shower (13) that have experienced different levels of hydrothermal alteration (14). The meteorite is an ungrouped type 2 carbonaceous chondrite (it has affinities to both $\mathrm{Cl}$ and $\mathrm{CM}$ meteorites) consisting of chondrules set in a fine-grained matrix that is dominated by serpentine and saponite clay minerals (15), and has been linked to the primitive D-type asteroids (16). Lithological variability on the scale of individual stones may be attributable to different conditions of alteration and/or impact brecciation (15). Tagish Lake contains a high concentration of organic matter, nearly $3 w t \%$ (17). An unusual distribution of soluble organic compounds that are dominated by carboxylic and sulfonic acids, with only trace (part-perbillion) levels of amino acids, has previously been reported for the Tagish Lake meteorite, suggesting a distinct pathway of organic synthesis compared to $\mathrm{Cl}$ and $\mathrm{CM}$ meteorites (18-19). Sub-micrometer-scale carbonaceous globules that are often substantially enriched in ${ }^{15} \mathrm{~N}$ and $\mathrm{D}$, and are thought to have formed in the ISM or the cold outer solar nebula, were previously identified in Tagish Lake $(5,20)$, demonstrating the preservation of such material in spite of parent body alteration.

Terrestrial contamination and modification, both abiotic and biotic, are perennial concerns in the study of meteorite organics. The first Tagish Lake meteorite specimens fell on a frozen lake, were collected without hand contact within a few days of the fall and have been kept frozen ever since (21), providing an opportunity for the study of organic matter in a pristine meteorite sample. Much of what is known about the Tagish Lake meteorite derives from studies of this pristine material (e.g., 18, 22). However, only a handful of the 48 pristine stones have been examined in detail (21). We selected four specimens from among these stones on the basis of their macroscopic properties, in order to carry out a systematic study of the variations in organic matter in this meteorite and to test whether variations in IOM or SOM correlate with petrologic differences. We processed subsamples of each of the four specimens ( $5 \mathrm{~b}$ - mass 4.3 $\mathrm{g}, 11 \mathrm{~h}-6.2 \mathrm{~g}, 11 \mathrm{i}-4.7 \mathrm{~g}$ and $11 \mathrm{v}-5.6 \mathrm{~g}$ ) in parallel, providing extracts for the analysis of SOM 
and IOM separates, material for X-ray diffraction (XRD), and polished mounts for microbeam analyses (13).

All four specimens are composed of olivine- and pyroxene-bearing chondrules and chondrule-like objects, compact lithic fragments, and isolated olivine or pyroxene grains, set in a fine-grained porous matrix dominated by clays, sulphides, magnetite, and carbonates. Based on the relative proportions of porous matrix and framboidal magnetite (15), and the increasing replacement of chondrule glass by phyllosilicates (23), the degree to which the specimens have undergone aqueous alteration is in the order $5 \mathrm{~b}<11 \mathrm{~h}<<11 \mathrm{i}$. Specimen $11 \mathrm{v}$, which consists of disaggregated material collected from the lake ice surface, is heterogeneous on the microscale, comprising clasts whose petrologic characteristics cover the range seen in the other three specimens. The macroscopic differences among the specimens are attributable to the proportions of the various components, as well as matrix grain size. For example, $11 \mathrm{i}$, which is very dark and tends to shed a residue of black dust, has a lower proportion of chondrules and a smaller average matrix grain size $(<5 \mu \mathrm{m})$.

Isotopic and chemical analyses of bulk IOM separates from each of the four specimens (Table 1, Fig. 1a) show that the largest variations are in the $\mathrm{H} / \mathrm{C}$ ratios and $\mathrm{H}$ isotopic compositions ( $\delta \mathrm{D})$; variations in $\mathrm{N}$ isotopic compositions and in $\mathrm{C}$ in IOM as a proportion of the rock are negligible. $\mathrm{C}$ isotopic compositions show a small but substantial decrease in the order $5 \mathrm{~b}$ $>11 \mathrm{~h}>11 \mathrm{i} \sim 11 \mathrm{v}$ (Table 1). The variations in $\mathrm{H} / \mathrm{C}$ and $\delta \mathrm{D}$ observed in IOM in these specimens span almost the entire range found amongst the different carbonaceous chondrite groups (Fig. 1a). This lends credence to the suggestion that the variation in IOM elemental and isotopic compositions found in chondrites is the result of parent body modification of a common precursor $(8)$. Furthermore, there is a linear correlation between $\mathrm{H} / \mathrm{C}$ ratios and $\delta \mathrm{D}$ values (Fig. 1). Solid state ${ }^{13} \mathrm{C}$ and ${ }^{1} \mathrm{H}$ nuclear magnetic resonance spectroscopy and carbon $\mathrm{X}$-ray Absorption Near-edge Spectroscopy [C-XANES, (24)] (13) indicate that the decrease in $\mathrm{H} / \mathrm{C}$ ratio is accompanied by an increase in the proportion of aromatic $\mathrm{C}$ in the IOM as well as a considerable increase in aromatic substitution, likely aromatic condensation (13). The change in $\mathrm{H} / \mathrm{C}$ was not accompanied by a substantial loss of $\mathrm{C}$ (Table 1), which may indicate that the aliphatic 
component in Tagish Lake was converted into aromatic carbon, while undergoing $\mathrm{H}$ isotopic exchange with the altering fluid and/or preferential $\mathrm{D}$ loss. This apparently facile transformation is unexpected. It is most likely caused by hydrothermal alteration, as is observed in experiments involving hydrous pyrolysis or reaction with water at elevated temperature and pressure $(11,25)$, and differs from the scenario where aliphatic carbon is selectively removed through reaction with an oxidant $(20)$.

High-spatial-resolution secondary ion mass spectroscopic (SIMS) measurements reveal that the isotopic differences observed in bulk IOM residues extend to sub-micrometer scales. IOM from sample $5 b$ shows not only a higher average $\mathrm{D} / \mathrm{H}$ ratio, but also a much higher proportion of very D-rich sub- $\mu \mathrm{m}$ sized isotopic hotspots (Fig. $1 \mathrm{~b}$ ) with more extreme $\mathrm{D} / \mathrm{H}$ ratios than those from $11 \mathrm{v}$ (maximum $\delta \mathrm{D} \sim 20,000 \%$ in $5 \mathrm{~b}$ vs. $7,000 \%$ in $11 \mathrm{v}$ ). These observations suggest that parent body alteration has substantially removed $\mathrm{D}$, decreasing the $\mathrm{D} / \mathrm{H}$ ratio on all spatial scales and reducing the number of hotspots. Similar variations in D enrichments and abundances between chondrites have been observed before, but never in a single chondrite. In contrast, the $\mathrm{N}$ isotopic distributions are similar except that $5 \mathrm{~b}$ contains about twice the number density of ${ }^{15} \mathrm{~N}$ hotspots (with $\delta^{15} \mathrm{~N}$ in both residues up to $\sim 800 \%$ ). This difference in behavior of $\mathrm{H}$ and $\mathrm{N}$ isotopes supports observations in previous studies that $\mathrm{D}$ and ${ }^{15} \mathrm{~N}$ enrichments in IOM tend to be decoupled (5). Isotopic hotspots are, in many cases, associated with carbonaceous nanoglobules $(5,20)$. Transmission electron microscope (TEM) examinations indicate that IOM from sample $5 \mathrm{~b}$ has a significantly higher fraction (7.5\%) of nanoglobules than does IOM from $11 \mathrm{v}(0.9 \%)$ (13). C-XANES (24) indicates the presence of two chemical classes of nanoglobules, one with a $\mathrm{C}$ functional group distribution similar to non-globular IOM and one dominated by aromatic functionality (13). Aromatic-type nanoglobule spectra are seen in a higher fraction of nanoglobules from $11 \mathrm{v}$ compared to $5 \mathrm{~b}$ (50\% versus $20 \%, 13)$. Taken together, the SIMS, TEM and XANES results suggest that ${ }^{15} \mathrm{~N}$-rich nanoglobules have been preferentially destroyed in specimen $11 \mathrm{v}$ by hydrothermal alteration. Moreover, the higher fraction of highly aromatic nanoglobules in the more altered sample supports the conclusion from the bulk data that the alteration largely affects the aliphatic component of the IOM. 
Based on IOM results, the degree of alteration reflected by the Tagish Lake specimens is $5 \mathrm{~b}<11 \mathrm{~h}<11 \mathrm{i}<11 \mathrm{v}$, which is consistent with the order inferred petrologically. Within this context, we examined the results of the soluble organic matter analysis to determine whether the hydrothermal alteration has resulted in the formation, modification or destruction of soluble organic molecules, and to elucidate the relationship between IOM and SOM during the alteration.

Monocarboxylic acids (MCAs) dominate the water extracts of Tagish Lake. MCAs, such as formic and acetic acids, play essential roles in biochemistry (e.g., 11, 27, 28); higher homologues are the fatty acids that self-assemble into membrane-bound vesicles in meteorite extracts and are the possible precursors to cell membranes (e.g., 29). We identified 11 MCAs in all specimens, including most of the members of the homologous series of linear, saturated MCAs from $C_{1}$ to $C_{10}$. One or two branched isomers were detected in all specimens with the exception of $5 \mathrm{~b}$, in which 17 branched isomers were detected, in addition to the 11 linear MCAs. Numerous alkyl-substituted phenols were also found exclusively in $5 \mathrm{~b}$. While, as in previous studies, $\delta^{13} \mathrm{C}$ values are generally consistent with terrestrial values, MCA hydrogen isotopic compositions are D-enriched, consistent with an extraterrestrial origin (2): (as measured in 5b) $\delta \mathrm{D}$ (acetic) $247 \%, \delta \mathrm{D}$ (formic/propanoic) $708 \%, \delta \mathrm{D}$ (butanoic) $562 \%, \delta \mathrm{D}$ (isopentanoic) 697 $\%$ (13). The observed concentrations of these low molecular weight MCAs are unusually high relative to other studies of carbonaceous chondrites [including Tagish Lake, (18)], ranging from 42 to $250 \mathrm{ppm}$ for formic and acetic acid (13). We attribute these large concentrations to the preservation of the meteorite below $0{ }^{\circ} \mathrm{C}$ since its recovery, which has minimized loss of volatile organics, such as formic acid, as well as the specifics of the analytical methods (13). In nearly all specimens, the concentrations of the straight-chain MCAs decrease in a logarithmic manner as the carbon number increases, with the exception of $5 b$, in which the acetic acid concentration exceeds that of formic acid. The $\delta^{13} \mathrm{C}$ values of MCAs differ among the specimens (Fig. 2). All specimens have common $\delta^{13} \mathrm{C} \sim-20 \%$ for formic acid, and higher homologues approach a constant value of $\sim-25 \%$ (e.g., average nonanoic acid $=-26 \pm 2 \%$ ) with increasing $\mathrm{C}$ number. The largest differences are observed in acetic acid, which ranges from $+8 \%$ (11h) to - 
$36 \%$ (5b). Of particular note is specimen $11 \mathrm{~h}$, which shows a decrease in $\delta^{13} \mathrm{C}$ with increasing carbon number (Fig. 2).

The differences in MCAs among the Tagish Lake specimens may be explained by differing degrees of parent body modification. With the exception of formic acid, specimens $5 \mathrm{~b}$ and $11 \mathrm{~h}$ contain the highest concentrations of MCAs, two to ten times greater than concentrations in $11 \mathrm{i}$ and $11 \mathrm{v}(13)$, attributable to loss or destruction of these water soluble compounds during progressive parent body alteration. The high proportion of branched isomers in specimen $5 \mathrm{~b}$ suggest that it preserves a more primary suite of compounds (2). The MCA pattern for 11 h shows a trend of decreasing $\delta^{13} \mathrm{C}$ with increasing carbon number, comparable to results for Murchison (30). Whereas this trend has been attributed to the preservation of the signature of kinetically controlled carbon addition in MCA synthesis, which takes place in cold, interstellar or nebular environments (31), our results, which suggest that specimen $11 \mathrm{~h}$ is more altered than 5 b, imply that such a pattern may be a secondary signature. One possible explanation for the pattern in this case is the preferential exchange of MCA carboxyl carbon with inorganic carbon during hydrothermal processing, analogous to oil-prone source rocks on Earth (32). In Tagish Lake, the presence of carbonate $\delta^{13} \mathrm{C} \sim 67 \%$ (17) may provide a source of isotopically enriched carbonate for such exchange. Notably, formic acid concentration and $\mathrm{C}$ isotopic composition remain relatively constant among the specimens (13), which suggests that it is relatively unaffected by aqueous alteration $(10)$ and may be inherited from pre-accretionary material.

Amino acid concentrations and enantiomeric excesses in the Tagish Lake specimens provide further evidence of the influence of parent body aqueous alteration on SOM. We determined the distribution and enantiomeric abundances of the one- to six-carbon aliphatic amino acids found in extracts of specimens, $5 \mathrm{~b}, 11 \mathrm{~h}$ and $11 \mathrm{i}$ by ultra-performance liquid chromatography fluorescence detection and time-of-flight mass spectrometry (33). We measured stable carbon isotope analyses of the most abundant amino acids in $11 \mathrm{~h}$ with gas chromatography coupled with quadrupole mass spectrometry and isotope ratio mass spectrometry. The total abundances of amino acids decrease in the order $11 \mathrm{~h}(5.6 \mathrm{ppm})>5 \mathrm{~b}(0.9$ $\mathrm{ppm})>11 \mathrm{i}(0.04 \mathrm{ppm})$. The abundances of many amino acids in $11 \mathrm{i}$ were below the analytical 
detection limit $(<1 \mathrm{ppb})$ which is consistent with a much higher degree of alteration experienced by $11 \mathrm{i}$ compared to $11 \mathrm{~h}$ and $5 \mathrm{~b}$. The abundance of the non-protein amino acid $\alpha-$ aminoisobutyric acid ( $\alpha$-AIB) in specimen $11 \mathrm{~h}$ was $0.2 \mathrm{ppm}$, approximately 200 times higher than previously measured in two different Tagish Lake meteorite samples (18-19). Glycine is the most abundant amino acid in $11 \mathrm{~h}$ and has a carbon isotope value of $\delta^{13} \mathrm{C}=+19 \%$, which falls well outside the range for terrestrial organic carbon of -6 to $-40 \% 0(34)$, and is consistent with an extraterrestrial origin.

The enantiomeric ratios of alanine, $\beta$-amino- $n$-butyric acid, and isovaline in $11 \mathrm{~h}$ were racemic within uncertainties $(\mathrm{D} / \mathrm{L}=1)$ providing additional evidence of an extraterrestrial origin for these amino acids. In contrast to specimen $11 \mathrm{~h}$, non-racemic isovaline was detected in $5 \mathrm{~b}$ with an L-enantiomeric excess of $\sim 7 \%$, and no isovaline was identified in $11 \mathrm{i}$ above the detection limit. Though the mechanism of enrichment remains unclear, it has been previously shown that $\mathrm{L}$-isovaline enantiomeric excesses (ee) and the ratio of $\beta$-alanine to glycine both increase relative to the degree of aqueous alteration for many carbonaceous chondrite groups $(33,35)$. Although the data for specimen $11 \mathrm{i}$ relative to $11 \mathrm{~h}$ or $5 \mathrm{~b}$ fit this trend (Fig. 3), in detail the sequence of alteration for $5 \mathrm{~b}$ and $11 \mathrm{~h}$ based on these criteria suggest that $5 \mathrm{~b}$ is more altered than $11 \mathrm{~h}$, in contrast to the result from petrography and IOM. This result suggests that other factors may influence ee and $\beta$-alanine/glycine ratio that are apparent in Tagish Lake. The higher ratio of $\beta$ m alanine to glycine in $5 \mathrm{~b}(\sim 0.6)$ compared to $11 \mathrm{~h}(\sim 0.2)$ may be due to enhanced production of glycine during aqueous alteration of $11 \mathrm{~h}$ via reactions involving hydroxy acids known to be present in SOM (e.g., 36, 37). A study of L-isovaline ee in Murchison specimens showed a range of ee values from 0 to $15 \%$, roughly correlative with abundance of hydrated minerals in the samples, indicating the role of multiple, complex parent body synthetic processes in amino acid formation (38). The amino acids in Tagish Lake 11h, including ee and overall abundance, may therefore be interpreted as reflecting a secondary pulse of amino acid formation resulting from hydrothermal alteration on the Tagish Lake parent body, which overprinted any original ee with a racemic mixture.

Substantial heterogeneity is preserved within the Tagish Lake meteorite especially in terms of organic matter. The correlation between differences in organic matter properties and 
indicators of hydrothermal alteration indicates that the processes were active after accretion onto the parent body. In this scenario, chondritic components, including $\mathrm{D}$ - and ${ }^{15} \mathrm{~N}$-rich IOM that is best preserved in $5 \mathrm{~b}$, were accreted, along with (presumably) amino acid precursors. The $\alpha$ amino acids were likely produced during alteration on the Tagish Lake parent body, presumably by Strecker synthesis $(37,39)$, although other formation mechanisms for both $\alpha$ and other amino acids prior to their incorporation in the parent body have been suggested (40). Modest alteration may have produced light acetic acid and an initial complement of MCAs from IOM, by analogy with experiments $(11)$, as well as a slight ee in isovaline, to provide the SOM characteristics observed in $5 \mathrm{~b}$. These components were then modified on the parent body through further hydrothermal alteration, resulting in reduction of aliphatic character and $\mathrm{D} / \mathrm{H}$ in $\mathrm{IOM}$, exchange of isotopically heavy carbon with MCA carboxyl carbon, production of glycine, and a fresh influx of racemic amino acids, as represented by organic matter in $11 \mathrm{~h}$. By analogy with MCAs, exchange of isotopically heavy carbon with amino acid carboxyl carbon may explain the positive $\delta^{13} \mathrm{C}$ values of amino acids in $11 \mathrm{~h}$ (e.g., glycine). The decrease in IOM $\delta^{13} \mathrm{C}$ with degree of alteration (Table 1) is consistent with loss of isotopically lighter carbon, associated with aliphatics, e.g., MCAs in $11 \mathrm{i}$ and 11v. Further hydrothermal alteration resulted in further modification of IOM and decreases in overall concentration of MCAs in $11 \mathrm{i}$ and $11 \mathrm{v}$ and a nearly complete loss of amino acids in 11i. The conditions of hydrothermal alteration inferred by analogy with experiments, especially temperature $\left(\sim 300^{\circ} \mathrm{C}\right)(10-11,25)$, are at odds with the mineralogy and preservation of volatile organic compounds, which provide an upper limit of $\sim$ $150^{\circ} \mathrm{C}$ (e.g., 23). The Tagish Lake specimens may, therefore, have experienced alteration at lower temperatures than the experiments, with the more extensively altered samples having been subjected to longer periods of alteration, higher temperatures, and/or water/rock ratios (11).

\section{References and Notes}

1. E. R. D. Scott, A. N. Krot, in Treatise on Geochemistry, H. D. Holland, K. K. Turekian, Eds. (Elsevier, 2007), vol. 1, pp. 1-72.

2. I. Gilmour, in Treatise on Geochemistry, H. D. Holland, K. K. Turekian, Eds. (Elsevier, 2003), vol. 1, pp. $269-290$.

3. O. Botta, J. L. Bada, Surveys in Geophysics 23, 411 (2002).

4. J. R. Cronin, S. Pizzarello, D. P. Cruikshank, in Meteorites and the Early Solar System, J. F. Kerridge, M. S. Matthews, Eds. (University of Arizona Press, Tucson, 1988), pp. 819-857.

5. H. Busemann et al., Science 312, 727 (May, 2006). 
6. S. A. Sandford et al., Science 314, 1720 (Dec, 2006).

7. J. Duprat et al., Science 328, 742 (May, 2010).

8. C. M. O. D. Alexander, M. Fogel, H. Yabuta, G. D. Cody, Geochimica et Cosmochimica Acta 71, 4380 (2007).

9. C. F. Chyba, P. J. Thomas, L. Brookshaw, C. Sagan, Science 249, 366 (Jul, 1990).

10. Y. Huang, M. R. Alexandre, Y. Wang, Earth and Planetary Science Letters 259, 517 (2007).

11. Y. Oba, H. Naraoka, Meteoritics \& Planetary Science 41, 1175 (Aug, 2006).

12. L. Remusat, S. Derenne, F. Robert, H. Knicker, Geochimica et Cosmochimica Acta 69, 3919 (2005).

13. Information on materials and methods is available on Science Online.

14. Hydrothermal alteration occurred early in the history of the carbonaceous chondrite parent bodies owing to accumulation of the heat of radioactive decay, such that liquid water was transiently present and percolated through the mineral matrix. The evidence for this process is preserved in mineral alterations. Furthermore, in the interior of the parent body the temperature and pressure can rise high enough to produce hydropyrolysis of organic material.

15. M. E. Zolensky et al., Meteoritics \& Planetary Science 37, 737 (2002).

16. T. Hiroi, M. Zolensky, C. M. Pieters, Science 293, 2234 (2001).

17. M. M. Grady, A. B. Verchovsky, I. A. Franchi, I. P. Wright, C. T. Pillinger, Meteoritics \& Planetary Science 37, 713 (2002).

18. S. Pizzarello et al., Science 293, 2236 (2001).

19. G. Kminek, O. Botta, D. P. Glavin, J. L. Bada, Meteoritics \& Planetary Science 37, 697 (2002).

20. K. Nakamura-Messenger, S. Messenger, L. P. Keller, S. J. Clemett, M. E. Zolensky, Science 314, 1439 (Dec, 2006).

21. R. K. Herd, C. D. K. Herd, Lunar and Planetary Science XXXVIII, Abstract \#2347 (2007).

22. P. G. Brown et al., Science 290, 320 (13 October, 2000).

23. A. J. Brearley, in Treatise on Geochemistry, H. D. Holland, K. K. Turekian, Eds. (Elsevier, 2003), vol. 1, pp. 247-268.

24. A. L. D. Kilcoyne et al., Journal of Synchrotron Radiation 10, 125 (2003).

25. H. Yabuta, L. B. Williams, G. D. Cody, C. M. O. Alexander, S. Pizzarello, Meteoritics \& Planetary Science 42, 37 (Jan, 2007).

26. G. D. Cody, C. M. O. D. Alexander, Geochimica et Cosmochimica Acta 69, 1085 (2005).

27. M. C. Anguera et al., Journal of Biological Chemistry 281, 18335 (2006).

28. G. K. Smith, W. T. Mueller, L. J. Slieker, C. W. DeBrosse, S. J. Benkovic, Biochemistry 21, 2870 (1982).

29. D. Deamer, J. P. Dworkin, S. A. Sandford, M. P. Bernstein, L. J. Allamandola, Astrobiology 2, 371 (2002).

30. Y. S. Huang et al., Geochimica et Cosmochimica Acta 69, 5167 (2005).

31. G. Yuen, N. Blair, D. J. Desmarais, S. Chang, Nature 307, 252 (1984).

32. R. F. Dias, K. H. Freeman, M. D. Lewan, S. G. Franks, Geochimica et Cosmochimica Acta 66, 2755 (2002).

33. D. P. Glavin, J. P. Dworkin, Proc. Natl. Acad. Sci. U. S. A. 106, 5487 (Apr, 2009).

34. R. Bowen, in Isotopes in the Earth Sciences, R. Bowen, Ed. (Kluwer, New York, 1988), pp. 452469.

35. D. P. Glavin, M. P. Callahan, J. P. Dworkin, J. E. Elsila, Meteoritics \& Planetary Science 45, 1948 (2010).

36. J. R. Cronin, S. Pizzarello, S. Epstein, R. V. Krishnamurthy, Geochimica et Cosmochimica Acta 57, 4745 (Oct, 1993). 
37. E. T. Peltzer, J. L. Bada, G. Schlesinger, S. L. Miller, Adv. Space Sci. 4, 69 (1984).

38. S. Pizzarello, M. Zolensky, K. A. Turk, Geochimica et Cosmochimica Acta 67, 1589 (2003).

39. E. T. Peltzer, J. L. Bada, Nature 272, 443 (1978).

40. J. E. Elsila, J. P. Dworkin, M. P. Bernstein, M. P. Martin, S. A. Sandford, Astrophys. J. 660, 911 (May, 2007).

41. Where given, the errors are half the difference (standard error in the mean) between the compositions of two residues prepared from two separate aliquots of each specimen. Typically, the differences in elemental ratios and isotopic compositions are larger than the intrinsic measurement precisions. Where only one measurement was made, the uncertainties of the other samples are a guide to the likely uncertainties.

42. D.P. Glavin et al., Meteoritics \& Planetary Science 41, 889 (2006).

43. J.E. Elsila, D. P. Glavin, J. P. Dworkin, Meteoritics \& Planetary Science 44, 1323 (2009).

44. G. Docherty, V. Jones, R.P. Evershed, Rapid Communications in Mass Spectrometry 15, 730 (2001).

45. J. P. Bradley, L. P. Keller, K. L. Thomas, T. B. Vander Wood, D. E. Brownlee, Lunar and Planetary Science XXIV, Abstract \#1087 (1993).

46. Y. Wang, Y. Huang, C. M. O. D. Alexander, M. Fogel, G. Cody, Geochimica et Cosmochimica Acta $69,3711(2005)$.

47. M. J. Wooller et al., Journal of Paleolimnology 31, 267 (2004).

48. B. T. De Gregorio et al., Geochimica et Cosmochimica Acta 74, 4454 (Aug, 2010).

49. E. Jarosewich, J. Res. Nat. Inst. Standards and Tech. 107, 681 (2002).

50. J. T. Armstrong, Microbeam Anal. 4, 177 (May-Jun, 1995).

Funding for this study was provided by the Natural Sciences and Engineering Research Council of Canada, Alberta Innovates, NASA (Astrobiology, including Carnegie Institution Astrobiology and the Goddard Center for Astrobiology; Origins of Solar Systems; Cosmochemistry and Postdoctoral Programs), the U.S. Office of Naval Research, the Carnegie institution of Washington (CIW), Grant MacEwan University and the Carnegie Institution of Canada. The Canadian Institute for Advanced Research is thanked for hosting workshops that facilitated work on the MCAs. J. Kirby assisted with MCA analysis. R. Bowden carried out bulk IOM analyses. XANES data were acquired on the STXM at beamline 5.3.2.2 of the Advanced Light Source, which is supported by the Director of the Office of Science, Department of Energy, under Contract No. DE-AC02-05CH11231, and by a W.M. Keck Foundation grant to the CIW. Three anonymous reviewers are thanked for constructive comments that improved the manuscript.

\section{Supporting Online Material}

www.sciencemag.org

Materials and Methods

Figs. S1, S2, S3, S4, S5

Tables S1, S2, S3, S4 


\section{Figure Captions}

Figure 1. A. Plot of $\mathrm{H} / \mathrm{C}$, a measure of the degree of aliphatic character, against hydrogen isotopic composition for the Tagish Lake specimens, including data on Tagish Lake from previous work (8). Also shown are representative data from other chondrite groups after ( 8 ), including ordinary chondrites $(\mathrm{OC})$. For reference, the $\mathrm{H} / \mathrm{C}$ value of an aliphatic molecule with infinite chain length is 2 ; aromatic organic matter has a maximum $\mathrm{H} / \mathrm{C}=1$ (benzene), and approaches low values $(\sim 0.1)$ as the number of fused aromatic rings approaches infinity. B. Maps of $\delta \mathrm{D} / \mathrm{H}$ values of IOM separates from Tagish Lake specimens $5 \mathrm{~b}$ and $11 \mathrm{v}$, derived from $\mathrm{H}$ and D raster ion images acquired with a Cameca NanoSIMS 50L ion microprobe.

Figure 2. Carbon isotopic composition of monocarboxylic acids in the Tagish Lake meteorite. Uncertainties represent the standard deviation of three injections for each sample. For measurements with low amplitude (e.g., nonanoic or decanoic acid) we used a value of $4 \%$, which is based on the accuracy achieved for standards run with low concentrations. Also shown are the results from (31) for Murchison monocarboxylic acids. Symbol size reflects relative concentration (13).

Figure 3. L-isovaline enantiomeric excesses (bars) and $\beta$-Alanine/Glycine ratios (circles) in Tagish Lake meteorite specimens $11 \mathrm{~h}, 5 \mathrm{~b}$, and $11 \mathrm{i}$ (shown in yellow), compared with results from $\mathrm{Cl}$ (red), CM (green) and CR (blue) chondrites of differing degrees of aqueous alteration (data from 33). The percentage L-excess is defined as $\mathrm{L}_{\mathrm{e}}=\mathrm{L} \%-\mathrm{D} \%$, with a negative value corresponding to a D-excess. 
Table 1. Summary of results of IOM analysis of Tagish Lake specimens.

\begin{tabular}{|c|c|c|c|c|c|}
\hline Sample & Previous & $11 \mathrm{v}$ & $11 \mathrm{i}$ & $11 \mathrm{~h}$ & $5 b$ \\
\hline$C(w t \%)$ & $\sim 2$ & $1.77(9)$ & $1.82(4)$ & 1.86 & $1.6(3)$ \\
\hline $\overrightarrow{\mathrm{H} / \mathrm{C}}$ & 337 & $0.44(1)$ & $0.51(2)$ & .594 & $0.72(4)$ \\
\hline $\mathrm{N} / \mathrm{C}$ & $0.043(2)$ & $0.041(1)$ & $0.042(2)$ & 0.042 & $0.042(2)$ \\
\hline$\delta^{13} \mathrm{C}(\% 0)$ & $-14.2(1)$ & -13 & $-13.3(1)$ & -14.3 & $-14.7(2)$ \\
\hline$\delta^{15} N(\%)$ & $73(2)$ & $58(2)$ & $53(1)$ & 57 & $57(4)$ \\
\hline$\delta \mathrm{D}(\% 0)$ & $596(4)$ & $815(25)$ & $992(15)$ & 1470 & $1844(10)$ \\
\hline
\end{tabular}

Notes: See (4l). Previous data from (8), 